\title{
The Linda who skipped the logic classes
}

\author{
Moshe Szweizer and Rivka Schlagbaum \\ CBRE Group Inc., Global Research and Consulting, Auckland, New Zealand
}

(Dated: March 02, 2021) - doi: 10.31219/osf.io/k7t69

\begin{abstract}
The letter points to a logical mistake found in "The conjunction fallacy in probability judgment" published by Tversky and Kahneman. Currently, at least 5,100 research papers reference this work, and an entire field of associated studies has been created based on the paper. These works assume the correctness of the original publication and reproduce the error without due critical analysis.
\end{abstract}

In 1983 Tversky and Kahneman published a paper on "The conjunction fallacy in probability judgment". The experiments reported in this work involved a now-famous question concerning Linda, a highly educated person, followed by a list of statements that were to be evaluated for their respective probability. The exercise was framed as follows:

Linda is 31 years old, single, outspoken and very bright. She majored in philosophy. As a student, she was deeply concerned with issues of discrimination and social justice, and also participated in antinuclear demonstrations

Among other answers the following two were presented:

a) Linda is a bank teller

b) Linda is a bank teller and is active in the feminist movement

Statements a) and b) were to be ranked according to their respective probability.

It was subsequently discussed that the answer a) should be seen as more probable than b) due to b) being a logical product of two statements, one of which involved a). Thus, b) which is ("p" and "q") is less probable than "p" alone. Currently, at least 5,100 research papers reference this work, and an entire field of associated studies has been created.

It is quite disheartening to observe that an elementary logical error was committed by the authors and that this error has not been identified up till now.

The mistake was due to the authors not recognizing the problem was set as a conditional probability statement $\{(a)$ if "r" then "p"; (b) if "r" then ("p" and "q"), where "r" stands for "highly educated" $\}$. Thus, the introductory statement about Linda's education needs to be treated as a part of the answer to correctly evaluate each probability. It was a logical error to treat the statements a) and b) on their own and to apply logical arithmetic to them in separation from the introduction.

To make this abundantly clear, one may rephrase the statements as follows:

a) "Linda is highly educated and she works as a bank teller".

This statement has a low probability as one expects a highly educated person to have a well-paid job. The statement indicates severe underemployment and is challenged by the test participants who assign it a low probability.

b) "Linda is highly educated but she is also protesting against the current social order, and she works as a bank teller"

This second statement is more probable, due to Linda's stance which many employers would find problematic. In particular, it suggests that Linda may be difficult to manage. Thus, it is more probable that she works in a low-paid job. In this respect, it is correct that the test participants chose this option as more probable. Modern society expects an explanation when an undervalue is observed, and in this case, it was provided by pointing to Linda's outside of work activity.

It is very concerning that the paper's error has not been recognized for so long, and even more troubling that the error has been propagated so extensively through the refereed publishing system. 


\section{References:}

Tversky, A., \& Kahneman, D. (1983). Extensional versus intuitive reasoning: The conjunction fallacy in probability judgment. Psychological Review, 90, 293-315.

\section{To Cite this article}

\section{APA}

Schlagbaum, R., \& Szweizer, M. (2021, March 2). The Linda who skipped the logic classes. https://doi.org/10.31219/osf.io/k7t69

MLA

Schlagbaum, Rivka, and Moshe Szweizer. "The Linda Who Skipped the Logic Classes." OSF Preprints, 2 Mar. 2021. Web.

\section{Chicago}

Schlagbaum, Rivka, and Moshe Szweizer. 2021. "The Linda Who Skipped the Logic Classes." OSF Preprints. March 2. doi:10.31219/osf.io/k7t69. 